\title{
Strain-gradient homogenization: a bridge between the asymptotic expansion and quadratic boundary condition methods
}

\author{
Vincent Monchiet ${ }^{a}$, Nicolas Auffray ${ }^{\mathrm{a}}$, Julien Yvonnet $^{\mathrm{a}}$, \\ ${ }^{a}$ Université Paris-Est, Laboratoire Modélisation et Simulation Multi Echelle, \\ LMSME UMR8208 CNRS, 5 boulevard Descartes, 77454 Marne la Vallée Cedex, \\ France
}

\begin{abstract}
In this paper we deal with the determination of the strain gradient elasticity coefficients of composite material in the framework of the homogenization methods. Particularly we aim to eliminate the persistence of the strain gradient effects when the method based on quadratic boundary conditions is considered. Such type of boundary conditions is often used to determine the macroscopic strain gradient elastic coefficients but leads to contradictory results, particularly when a RVE is made up of a homogeneous material. The resulting macroscopic equivalent material exhibits strain gradient effects while it should be expected of Cauchy type. The present contribution is to provides new relationship to correct the approach based on the quadratic boundary condition. To this purpose, we start from the asymptotic homogenization approach, we establish a connection with the method based on quadratic boundary conditions and we highlight the correction required to eliminate the persistence of the strain gradient effects. An application to a composite with fibers is provided to illustrate the method.
\end{abstract}

Key words: Strain Gradient Elasticity, Homogenization, Quadratic Boundary Conditions, Asymptotic Expansion, Composite Material.

\footnotetext{
Email addresses: vincent.monchiet@u-pem.fr (Vincent Monchiet), nicolas.auffray@u-pem.fr (Nicolas Auffray), vincent.monchiet@u-pem.fr (Julien Yvonnet).
} 


\section{Introduction}

The derivation of strain gradient elasticity theory in the framework of homogenization approaches has been the subject of intense research during the last years but, in fact, has begun earlier with the works of Diener et al. [21, 22, 20]. Starting from the variational principles of Hashin-Shtrikmann [32, 33], Dederichs and Zeller [19] and Kroener and Koch [36] derived new bounds for the elastic coefficients when a random medium is subjected to non homogeneous mean fields. As a consequence the homogenized elastic coefficients depend on the wave vector of the mean field and, due to the emergence of internal lengths, the macroscopic behavior turns out to be non-local. The link with strain-gradient elasticity has been established later by [25] who assumed a polynomial representation for the mean field. By doing so, the overall behavior shows dependence on the gradient(s) of the macroscopic strain and, based on the usual energy principle, the authors derive the elastic coefficients of the Toupin [45] and Mindlin [37] strain-gradient model. However, such an approach only provides bounds for the higher-order elastic coefficients and not their exact values, and is restricted to some particular microstructures (examples can be found in $[23,24])$.

Strain gradient elasticity models have also been derived using asymptotic series expansion methods. Periodic homogenization approaches have been introduced and developed by Auriault and Sanchez-Palencia [5], Bensoussan et al. [10], Sanchez-Palencia [42], Suquet [44]... They are based on the introduction of a small parameter, the scale factor, and the determination of the solution by means of polynomial expansion series in power of this small parameter. By accounting for the second-order term of the series, Bakhvalov and Panasenko [8], Gambin and Kroener [29], Boutin [14], Triantafyllidis and Bardenhagen [47] found that the local solution exhibits a dependence with the macroscopic strain gradient (and also higher-order derivatives of the macroscopic strain gradient when accounting for higher-order local solutions). The connection with the Toupin and Mindlin strain gradient elasticity model has been really established by Smyshlyaev and Cherednichenko [43], Peerlings and Fleck [38], Tran et al. [46]. The strain gradient elasticity coefficients are computed by solving unit cell problems with Periodic Boundary Conditions (PBC). The first problem provides the fundamental solution and, by only keeping this term in the series expansion, the macroscopic description of the material remains of Cauchy type. Higher-order cell problems introduce the microstructural effects, i.e. the macroscopic description depends on an internal length scale that is characteristic of the microstructure and of the dependence of the elastic relationship with higher order derivatives of the macroscopic strain. The numerical implementation of the higher-order cell problems and the determination of strain-gradient elasticity coefficients have been already addressed in [38] using the Finite Element Method (FEM) or in [46] considering iterative schemes based on Fast Fourier Transform (FFT). It has been observed that 
when the scaling of elastic coefficients of the constituents have the appropriate order with respect to the scale factor, the effective material may exhibit strong strain gradient effects. This effect has been evidenced in the case of elastic composites reinforced by highly rigid slender inclusions by Pideri and Seppecher [40,39] and Boutin and Soubestre [16]. More recently, it has been demonstrated that the effective elastic energy of lattice materials having soft modes of deformation can be of generalized type [3]. Some general results have been obtained in $[2,1]$ combining asymptotic analysis and $\Gamma$-convergence method. However even if a method to determine the higher-order parameters was proposed in this last reference it fails when the first order elasticity is not degenerated.

Another attractive method for the computation of strain-gradient elasticity coefficients is based on the use of the Quadratic Boundary Conditions (QBC) on the Representative Volume Element (RVE). It generalizes the classic approach based on Uniform Strain Boundary Conditions (USBC) by applying a displacement that has a quadratic dependence with the position vector. The enforcing term is the macroscopic gradient of strain (or equivalent the macroscopic double gradient of displacement). The method is generally attributed to Besdo et al. [11, 12] but has been really introduced in the context of homogenization by Gologanu et al. [30] who derived the strain-gradient version of the Gurson model [31]. As already mentioned in [26], the results are however somewhat surprising in that the dominant effect of strain-gradient effects is not influenced by the void volume fraction or void spacing and the strain gradient effects persist even when the void volume fraction tends to zero. Indeed, the strain gradient effects are due to the presence of a microstructure at a lower scale and when the volume fraction tends to zero, the solid becomes homogeneous and no microstructural effects should be observed. Later, the method of Gologanu et al. [30] has been applied in the context of elasticity by Zybell et al. [49] with the same result: when radii of the inclusions tend to zero, the resulting microstructural effects still persist instead of vanishing as they should. The method has been later used in the case of elasticity and non linear constituents $[27,28,17,18,34,35]$ but in each case, the persistence of the strain gradient is retrieved for a homogeneous material at the local scale. This has been again reported in [48] who suggested that the method surely over evaluates the macroscopic energy density and probably exaggerates the strain gradient effects. It is important to notice that such observation does not concern the periodic homogenization based on asymptotic series expansion. Indeed, when the medium is homogeneous at the local scale, the source term in the second order cell problem, which depends only on the fluctuation, vanishes and the macroscopic behavior remains Cauchy elastic. Note that another approach based on QBC has been used by Bigoni and Drugan [13], Bacca et al. $[6,7]$. However their method is quite different in the sense that it is based on a small perturbation of the elastic properties and by approximating its effects on the macroscopic behavior by a strain gradient term. The approach is more related to the statistical method developed in [25]. 
The paper is organized as follows. To evidence the issue with the QBC based method, we start from the periodic homogenization with the asymptotic series expansion and we establish the connection between the two methods. To this end, we give a brief overview of the homogenization method based on asymptotic series expansion in section 3 . The first two unit cell problems, that lead to the reference solution and the first corrector, are detailed. In section 4 , using an appropriate change of variables, a bridge between the two homogenization procedures is established. In section 5, we discuss the results and we provide a new method to determine correctly the strain gradient elastic coefficients in the QBC based approach. Finally, in section 6, we propose an illustration for a composite with fibers. FEM computations with QBC are compared to FFT simulations for the problem with PBC.

\section{Notations}

PBC: Periodic Boundary Conditions.

QBC: Quadratic Boundary Conditions.

USBC: Uniform Strain Boundary Conditions.

RVE: Representative Volume Element.

FFT: Fast Fourier Transform.

\section{Tensorial notations:}

Vectors, second order and third order tensors are denoted by bold letters, usually lower case for microscopic quantities $\boldsymbol{u}$, and upper case for the associated macroscopic ones $\boldsymbol{U}$. Fourth- and higher-order tensors are denoted by blackboard letters such as $\mathbb{C}$.

$\odot_{n}$ stands for the generalized dot product between two tensors of order $\geq n$ over $n$ indices. For example, considering the second order tensors $\boldsymbol{A}$ and $\boldsymbol{B}$ we have $\boldsymbol{A} \odot_{2} \boldsymbol{B}=A_{i j} B_{i j}$ and for third order tensors $\boldsymbol{S}$ and $\boldsymbol{T}$ we have $\boldsymbol{S} \odot_{3} \boldsymbol{T}=S_{i j k} T_{i j k}$. The gradient operator with respect to the variable $\alpha$ is denoted by $\nabla_{\alpha}$ and the divergence operator with respect to $\alpha$ by $\operatorname{div}_{\alpha}$. By extension $\varepsilon_{\alpha}$ is the symmetric gradient operator defined as:

$$
\boldsymbol{\varepsilon}_{\alpha}(\boldsymbol{v})=\frac{1}{2}\left(\nabla_{\alpha} \boldsymbol{v}+\nabla_{\alpha}^{T} \boldsymbol{v}\right)
$$

The averaging of a tensorial quantity $\boldsymbol{T}$ over a spatial domain $V$ is denoted by:

$$
<\boldsymbol{T}>_{V}=\frac{1}{|V|} \int_{V} \boldsymbol{T} \mathrm{d} V
$$

where $\left(\mathbb{A}^{i}\right)^{T}$ represents the transpose of $\mathbb{A}^{i}$ obtained by the permutation of the two first and the $i+2$ last indices: $\left(\mathbb{A}^{0}\right)_{i j p q}^{T}=A_{p q i j}^{0}$ and $\left(\mathbb{A}^{1}\right)_{i j p q r}^{T}=A_{p q r i j}^{1}$. 


\section{Brief review of the periodic homogenization approach}

Asymptotic methods in periodic homogenization, initially introduced by SanchezPalencia [41], Bensoussan et al. [10], have been later considered by Bakhvalov and Panasenko [8], Gambin and Kroener [29], Boutin [14] to evaluate the deviation from the standard elastic description. The rigorous convergence of the method in the classical situation has been demonstrated by Allaire in [4]. In this section, only the main and necessary results are recalled, all the details can be found in the above cited papers. Consider the composite structure occupying an open set $\Omega$ of $\mathbb{R}^{d}$ whose characteristic length is $L$. As a periodic composite material, the (macro) domain $\Omega$ is supposed to be paved with unit cells. The domain of the elementary cell is denoted by $\omega$ and $h$ indicates the characteristic length of this underlying microstructure (see Fig. 1).

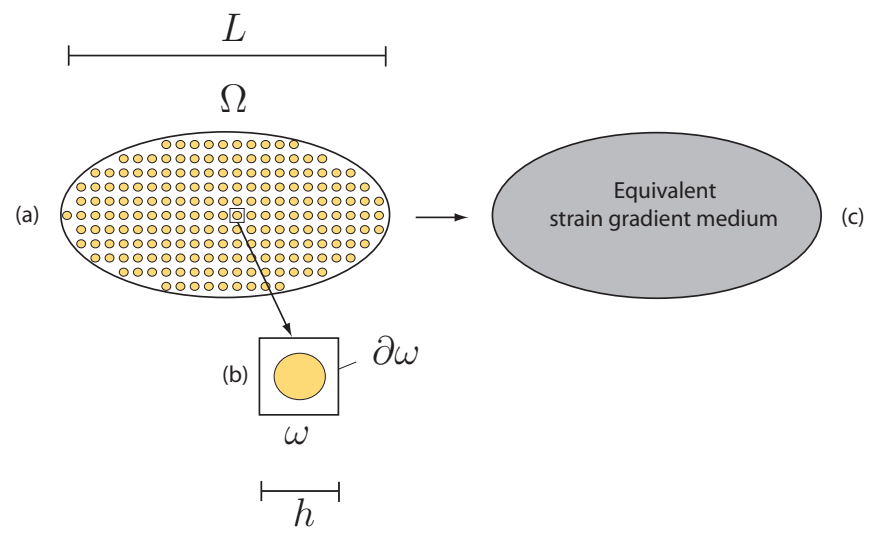

Fig. 1. (a) Heterogeneous structure, (b) unit cell, (c) Equivalent homogeneous strain gradient medium

The main feature of the asymptotic expansion method consist in the introduction of two space variables $\boldsymbol{x}=\boldsymbol{X} / L$ and $\boldsymbol{y}=\boldsymbol{X} / h$ where $L$ and $h$ are two characteristic lengths of the macro and the microstructure. The separation ratio between the two scales of the problem is measured by the scale factor $\epsilon=h / L$. The elastostatic problem can be formulated as follows:

$$
\left\{\begin{array}{l}
\operatorname{div}(\boldsymbol{\sigma}(\boldsymbol{x}, \boldsymbol{y}))=\boldsymbol{f}(\boldsymbol{x}, \boldsymbol{y}) \\
\boldsymbol{\sigma}(\boldsymbol{x}, \boldsymbol{y})=\mathbb{C}(\boldsymbol{y}): \boldsymbol{\varepsilon}(\boldsymbol{x}, \boldsymbol{y})
\end{array}\right.
$$

with some boundary conditions on $\partial \Omega$. In this formulation it is worth noting that the elasticity tensor is a periodic function of $\boldsymbol{y}$ with period $h$, meaning that the elastic properties are considered as macroscopically homogeneous (no global dependence on $\boldsymbol{x}$ ) but varying at the scale of the microstructure. The principle of the asymptotic method consists to search the solution in term of 
power series in $\epsilon$ :

$$
\boldsymbol{u}(\boldsymbol{x}, \boldsymbol{y})=\sum_{n=0}^{\infty} \epsilon^{n} \boldsymbol{u}^{n}(\boldsymbol{x}, \boldsymbol{y})
$$

in which each term $\boldsymbol{u}^{n}(\boldsymbol{x}, \boldsymbol{y})$ is periodic with respect to $\boldsymbol{y}$ with period $h$. It involves the following series expansions for the strain and stress fields:

$$
\begin{aligned}
& \boldsymbol{\varepsilon}(\boldsymbol{x}, \boldsymbol{y})=\epsilon^{-1} \varepsilon_{y}\left(\boldsymbol{u}^{0}(\boldsymbol{x}, \boldsymbol{y})\right)+\sum_{n=0}^{\infty} \epsilon^{n}\left[\varepsilon_{x}\left(\boldsymbol{u}^{n}(\boldsymbol{x}, \boldsymbol{y})\right)+\boldsymbol{\varepsilon}_{y}\left(\boldsymbol{u}^{n+1}(\boldsymbol{x}, \boldsymbol{y})\right)\right] \\
& \boldsymbol{\sigma}(\boldsymbol{x}, \boldsymbol{y})=\sum_{n=0}^{\infty} \epsilon^{n} \boldsymbol{\sigma}^{n}(\boldsymbol{x}, \boldsymbol{y})
\end{aligned}
$$

It has been demonstrated that the displacement $\boldsymbol{u}^{0}(\boldsymbol{x}, \boldsymbol{y})$ is independent of $\boldsymbol{y}$ and only depends on $\boldsymbol{x}$. It can be interpreted as a macroscopic displacement $\boldsymbol{U}(\boldsymbol{x})$. The higher-order terms $\boldsymbol{u}^{n \geq 1}(\boldsymbol{x}, \boldsymbol{y})$, which are the fluctuations due to the local heterogeneity, are determined by solving elementary cell problems over $\omega$. For instance, the displacement $\boldsymbol{u}^{1}(\boldsymbol{x}, \boldsymbol{y})$ is solution of the set of equations:

$$
\left\{\begin{array}{l}
\operatorname{div}_{y}\left(\boldsymbol{\sigma}^{0}(\boldsymbol{x}, \boldsymbol{y})\right)=0 \\
\boldsymbol{\sigma}^{0}(\boldsymbol{x}, \boldsymbol{y})=\mathbb{C}(\boldsymbol{y}): \varepsilon^{0}(\boldsymbol{x}, \boldsymbol{y}) \\
\boldsymbol{\varepsilon}^{0}(\boldsymbol{x}, \boldsymbol{y})=\boldsymbol{\varepsilon}_{y}\left(\boldsymbol{u}^{1}(\boldsymbol{x}, \boldsymbol{y})\right)+\boldsymbol{E}(\boldsymbol{x}) \\
\boldsymbol{\varepsilon}_{y}\left(\boldsymbol{u}^{1}(\boldsymbol{x}, \boldsymbol{y})\right)=\frac{1}{2}\left(\nabla_{y} \boldsymbol{u}^{1}(\boldsymbol{x}, \boldsymbol{y})+\nabla_{y}^{T} \boldsymbol{u}^{1}(\boldsymbol{x}, \boldsymbol{y})\right)
\end{array}\right.
$$

with the following periodic boundary conditions over the unit cell $\omega$

$$
\boldsymbol{u}^{1} \text { y-periodic, } \boldsymbol{\sigma}^{0} \cdot \boldsymbol{n} \text { y-antiperiodic }
$$

The solution at first order linearly depends on the applied macroscopic strain $\boldsymbol{E}(\boldsymbol{x})=\frac{1}{2}\left(\nabla_{x} \boldsymbol{U}(\boldsymbol{x})+\nabla_{x}^{T} \boldsymbol{U}(\boldsymbol{x})\right)$. The localization rule is defined in the form:

$$
\varepsilon^{0}(\boldsymbol{x}, \boldsymbol{y})=\mathbb{A}^{0}(\boldsymbol{y}) \odot_{2} \boldsymbol{E}(\boldsymbol{x})
$$

Higher-order terms of the series expansion account for the microstructural effects which are expected when the scale factor $\epsilon$ is not small enough. By neglecting all higher-order terms of the series, the macroscopic description is of Cauchy type.

Consider the first higher-order term of the series given by the displacement $\boldsymbol{u}^{2}$. It is determined by solving the following unit cell problem: 


$$
\left\{\begin{array}{l}
\operatorname{div}_{y}\left(\boldsymbol{\sigma}^{1}(\boldsymbol{x}, \boldsymbol{y})\right)+\operatorname{div}_{x}\left(\boldsymbol{\sigma}^{0}(\boldsymbol{x}, \boldsymbol{y})-<\boldsymbol{\sigma}^{0}(\boldsymbol{x}, \boldsymbol{y})>_{\omega}\right)=0 \\
\boldsymbol{\sigma}^{1}(\boldsymbol{x}, \boldsymbol{y})=\mathbb{C}(\boldsymbol{y}): \varepsilon^{1}(\boldsymbol{x}, \boldsymbol{y}) \\
\boldsymbol{\varepsilon}^{1}(\boldsymbol{x}, \boldsymbol{y})=\boldsymbol{\varepsilon}_{y}\left(\boldsymbol{u}^{2}(\boldsymbol{x}, \boldsymbol{y})\right)+\boldsymbol{\varepsilon}_{x}\left(\boldsymbol{u}^{1}(\boldsymbol{x}, \boldsymbol{y})\right) \\
\boldsymbol{\varepsilon}_{y}\left(\boldsymbol{u}^{2}(\boldsymbol{x}, \boldsymbol{y})\right)=\frac{1}{2}\left(\nabla_{y} \boldsymbol{u}^{2}(\boldsymbol{x}, \boldsymbol{y})+\nabla_{y}^{T} \boldsymbol{u}^{2}(\boldsymbol{x}, \boldsymbol{y})\right) \\
\boldsymbol{\varepsilon}_{x}\left(\boldsymbol{u}^{1}(\boldsymbol{x}, \boldsymbol{y})\right)=\frac{1}{2}\left(\nabla_{x} \boldsymbol{u}^{1}(\boldsymbol{x}, \boldsymbol{y})+\nabla_{x}^{T} \boldsymbol{u}^{1}(\boldsymbol{x}, \boldsymbol{y})\right)
\end{array}\right.
$$

where the periodic boundary conditions (8) are still considered for the displacement $\boldsymbol{u}^{2}$ and the stress $\boldsymbol{\sigma}^{1}$. The loading parameters are the solution of the unit cell problem at previous order: the displacement $\boldsymbol{u}^{1}$ and the stress $\boldsymbol{\sigma}^{0}$. Since these fields linearly depend on the applied macroscopic strain, when we apply the differential operator " $\operatorname{div}_{x}()$ " and " $\varepsilon_{x}()$ ", these terms linearly depend on the macroscopic gradient of strain $\nabla_{x} \boldsymbol{E}$. As a consequence, the displacement field $\boldsymbol{u}^{2}$ linearly depends on the macroscopic gradient of strain $\nabla_{x} \boldsymbol{E}$. The localization rule for the first corrector can be read in the form:

$$
\varepsilon^{1}=\mathbb{A}^{1}(\boldsymbol{y}) \odot_{3} \nabla_{x} \boldsymbol{E}(\boldsymbol{x})
$$

An energy based upscaling approach can be employed to determine the Mindlin strain gradient elasticity tensor following Tran et al. [46]. For instance, if only the two first terms are kept in the series expansion for the strain, the latter reads $\varepsilon=\varepsilon^{0}+\epsilon \varepsilon^{1}$ in which $\varepsilon^{0}$ and $\varepsilon^{1}$ linearly depend on the macroscopic strain and gradient of strain respectively (see Eq. (9) and Eq. (11)). As a consequence the macroscopic elastic energy $W$ computed with the first two terms of the series reads:

$$
\begin{aligned}
W & =\frac{1}{2}\langle\boldsymbol{\sigma}: \boldsymbol{\varepsilon}\rangle_{\omega} \\
& =\frac{1}{2} \boldsymbol{E} \odot_{2} \mathbb{C}^{0,0} \odot_{2} \boldsymbol{E}+\epsilon \boldsymbol{E} \odot_{2} \mathbb{C}^{0,1} \odot_{3} \nabla_{x} \boldsymbol{E}+\frac{1}{2} \epsilon^{2} \nabla_{x} \boldsymbol{E} \odot_{3} \mathbb{C}^{1,1} \odot_{3} \nabla_{x} \boldsymbol{E}
\end{aligned}
$$

in which the tensors $\mathbb{C}^{i, j}$ are defined by:

$$
\mathbb{C}^{i, j}=<\left(\mathbb{A}^{i}(\boldsymbol{y})\right)^{T} \odot_{2} \mathbb{C}(\boldsymbol{y}) \odot_{2} \mathbb{A}^{j}(\boldsymbol{y})>_{\omega}
$$

In the expression of the macroscopic elastic energy $W$, the macroscopic gradient of strain $\nabla_{x} \boldsymbol{E}$ can be replaced by $L \nabla_{\chi} \boldsymbol{E}$ where $\boldsymbol{\chi}=\boldsymbol{x} L$ is the true macroscopic space variable ( $\boldsymbol{x}$ being the dimensionless macroscopic space variable). Therefore, the macroscopic energy density becomes: 


$$
\begin{gathered}
W=\frac{1}{2} \boldsymbol{E} \odot_{2} \mathbb{C}^{0,0} \odot_{2} \boldsymbol{E}+h \boldsymbol{E} \odot_{2} \mathbb{C}^{0,1} \odot_{3} \nabla_{\chi} \boldsymbol{E} \\
+\frac{1}{2} h^{2} \nabla_{\chi} \boldsymbol{E} \odot_{3} \mathbb{C}^{1,1} \odot_{3} \nabla_{\chi} \boldsymbol{E}
\end{gathered}
$$

which depends on the size $h$ which defines the period of the microstructure. It must be pointed out that the present method is based on a truncated description that is certainly questionable. By keeping only the first two terms of the series, we just approximate the solution, and this approximation is quite uncontrolled. The reader could refer to the discussion in [15].

\section{Reformulation of cell problems with linear and quadratic bound- ary condition}

\subsection{First order problem}

Consider the first order problem, defining the displacement $\boldsymbol{u}^{1}$ (see equations (7) with (8)). Let us apply the following change of variable:

$$
\boldsymbol{u}^{1}=\boldsymbol{u}^{L}-\boldsymbol{E}(\boldsymbol{x}) \cdot \boldsymbol{y}, \quad \boldsymbol{\sigma}^{0}=\boldsymbol{\sigma}^{L}
$$

With this definition, the displacement contains (i) the periodic part $\boldsymbol{u}^{L}$ due to the local heterogeneities (the latter vanishes for a homogeneous material at the local scale) and (ii) a linear displacement $\boldsymbol{E}(\boldsymbol{x}) \cdot \boldsymbol{y}$. With this change of variable, the first local problem becomes:

$$
\left\{\begin{array}{l}
\operatorname{div}_{y}\left(\boldsymbol{\sigma}^{L}\right)=0 \\
\boldsymbol{\sigma}^{L}=\mathbb{C}(\boldsymbol{y}): \boldsymbol{\varepsilon}_{y}\left(\boldsymbol{u}^{L}\right)
\end{array}\right.
$$

The usual homogenization problem with the uniform strain boundary condition is retrieved if we replace the periodic condition for $\boldsymbol{u}^{1}$ by the condition $\boldsymbol{u}^{1}=0$ on the boundary $\partial \omega$. This leads to:

$$
\boldsymbol{u}^{L}=\boldsymbol{E} \cdot \boldsymbol{y}
$$

that is also called linear condition in the sense that $\boldsymbol{u}^{L}$ linearly depends on the space variable $\boldsymbol{y}$. The solution of the problem can be read:

$$
\varepsilon^{L}=\mathbb{A}^{L}(\boldsymbol{y}) \odot_{2} \boldsymbol{E}
$$

where $\mathbb{A}^{L}(\boldsymbol{y})$ is the fourth order localisation tensor. 


\subsection{Second order problem}

We aim to provide the same analogy for the second order problem. Let us start from the set of Eqs. (10). Let us recall that the stress field $\boldsymbol{\sigma}^{0}$ is divergence-free with respect to the coordinate $\boldsymbol{y}$ (see Eq. (7)). As a consequence, this stress satisfies:

$$
\frac{\partial \sigma_{i j}^{0}}{\partial x_{j}}=\frac{\partial}{\partial y_{j}}\left[\frac{\partial \sigma_{i j}^{0}}{\partial x_{k}} y_{k}\right]
$$

Let us introduce the stress $\boldsymbol{\sigma}^{Q}$ defined by:

$$
\boldsymbol{\sigma}^{Q}=\boldsymbol{\sigma}^{1}+\frac{\partial \boldsymbol{\sigma}^{0}}{\partial x_{k}} y_{k}
$$

Owing to Eq. (19) and the first equation in (10), we deduce that:

$$
\operatorname{div}_{y}\left(\boldsymbol{\sigma}^{Q}\right)=\operatorname{div}_{x}\left(<\boldsymbol{\sigma}^{L}>_{V}\right)
$$

Note that the term on the right-end of the equality in Eq. (21) is constant at the local scale and can be interpreted as a constant body force.

Now, replacing in Eq. (20) the expressions of $\boldsymbol{\sigma}^{0}$ (cf. second equation in (7)) and $\boldsymbol{\sigma}^{1}$ (cf. second equation in (10)), we obtain:

$$
\boldsymbol{\sigma}^{Q}=\mathbb{C}(\boldsymbol{y}):\left(\boldsymbol{\varepsilon}_{y}\left(\boldsymbol{u}^{2}\right)+\boldsymbol{\varepsilon}_{x}\left(\boldsymbol{u}^{1}\right)\right)+\frac{\partial}{\partial x_{k}}\left[\mathbb{C}(\boldsymbol{y}):\left(\boldsymbol{\varepsilon}_{y}\left(\boldsymbol{u}^{1}\right)+\boldsymbol{E}(\boldsymbol{x})\right)\right] y_{k}
$$

Let us now use in the above equation the following property:

$$
\frac{\partial \boldsymbol{\varepsilon}_{y}\left(\boldsymbol{u}^{1}\right)}{\partial x_{k}} y_{k}=\boldsymbol{\varepsilon}_{y}\left(\frac{\partial \boldsymbol{u}^{1}}{\partial x_{k}} y_{k}\right)-\boldsymbol{\varepsilon}_{x}\left(\boldsymbol{u}^{1}\right)
$$

It leads to:

$$
\boldsymbol{\sigma}^{Q}=\mathbb{C}(\boldsymbol{y}):\left[\boldsymbol{\varepsilon}_{y}\left(\boldsymbol{u}^{2}+\frac{\partial \boldsymbol{u}^{1}}{\partial x_{k}} y_{j}\right)+\nabla_{x} \boldsymbol{E}(\boldsymbol{x}) \cdot \boldsymbol{y}\right]
$$

We also introduce the displacement $\boldsymbol{u}^{Q}$ defined by:

$$
\boldsymbol{u}^{Q}=\boldsymbol{u}^{2}+\frac{\partial \boldsymbol{u}^{1}}{\partial x_{k}} y_{k}+\frac{1}{2} \nabla_{x}^{2} \boldsymbol{U}:(\boldsymbol{y} \otimes \boldsymbol{y})
$$

where $\nabla_{x}^{2} \boldsymbol{U}$ is the double gradient of macroscopic displacement. The components of $\nabla_{x}^{2} \boldsymbol{U}$ are related to that of the macroscopic gradient of strain $\nabla_{x} \boldsymbol{E}$ by:

$$
E_{i j, k}=\frac{1}{2}\left(U_{i, j k}+U_{j, i k}\right) \Leftrightarrow U_{i, j k}=E_{i k, j}+E_{i j, k}-E_{j k, i}
$$

where $E_{i j, k}$ are the components of the macroscopic gradient of strain $\nabla_{x} \boldsymbol{E}$ and $U_{i, j k}$ are the components of the double gradient of the macroscopic displacement, $\nabla_{x}^{2} \boldsymbol{U}$. The components $U_{i, j k}$ are invariant by any permutation of 
the indices $j$ and $k$, while $E_{i j, k}$ is invariant by any permutation of the indices $i$ and $j$. Both tensors depend on 18 independent coefficients in $\mathbb{R}^{3}$, and 6 in $\mathbb{R}^{2}$.

Owing to Eq. (25), Eq. (24) can be written as:

$$
\boldsymbol{\sigma}^{Q}=\mathbb{C}(\boldsymbol{y}): \boldsymbol{\varepsilon}_{y}\left(\boldsymbol{u}^{Q}\right)
$$

Following the previous section we now aim to identify the relation between the second order problem coming from the asymptotic homogenization and the problem with the quadratic boundary condition (QBC). To this end, we replace the periodic boundary conditions for the displacements $\boldsymbol{u}^{1}$ and $\boldsymbol{u}^{2}$ by the conditions $\boldsymbol{u}^{1}=0$ and $\boldsymbol{u}^{2}=0$ on $\partial \omega$. Therefore, we observe that the displacement $\boldsymbol{u}^{Q}$ satisfies:

$$
\boldsymbol{u}^{Q}=\frac{1}{2} \nabla_{x}^{2} \boldsymbol{U}:(\boldsymbol{y} \otimes \boldsymbol{y}) \quad \forall \boldsymbol{y} \in \partial \omega
$$

To sum up, the second-order problem can be summarized as:

$$
\begin{array}{rr}
\operatorname{div}_{y}\left(\boldsymbol{\sigma}^{Q}\right)=\operatorname{div}_{x}\left(<\boldsymbol{\sigma}^{L}>_{V}\right) & \forall \boldsymbol{y} \in \omega \\
\boldsymbol{\sigma}^{Q}=\mathbb{C}(\boldsymbol{y}): \varepsilon_{y}\left(\boldsymbol{u}^{Q}\right) & \forall \boldsymbol{y} \in \omega \\
\boldsymbol{u}^{Q}=\frac{1}{2} \nabla_{x}^{2} \boldsymbol{U}:(\boldsymbol{y} \otimes \boldsymbol{y}) & \forall \boldsymbol{y} \in \partial \omega
\end{array}
$$

with $\boldsymbol{\sigma}^{L}$ computed from the first-order problem (with the linear boundary condition).

This problem is almost equivalent to the one used by many authors to evaluate the strain gradient elastic properties $[27,17,18,34,35]$ but differs by the presence of the body force term $\left.\operatorname{div}_{x}\left(<\boldsymbol{\sigma}^{L}\right\rangle_{V}\right)$ in the equilibrium equation (21).

The localization rule associated with the problem corresponding to the QBC is:

$$
\varepsilon^{Q}=\mathbb{A}^{Q}(\boldsymbol{y}) \odot_{3} \nabla_{x} \boldsymbol{E}
$$

\section{Discussion}

Let us first give the relation that exists between the strain field $\boldsymbol{\varepsilon}_{y}\left(\boldsymbol{u}^{Q}\right)$ computed with the QBC and the first corrector $\varepsilon^{1}$. For the sake of simplicity, $\varepsilon^{L}$ will stand for $\varepsilon_{y}\left(\boldsymbol{u}^{L}\right)$ and $\varepsilon^{Q}$ for $\varepsilon_{y}\left(\boldsymbol{u}^{Q}\right)$. The first corrective term for the strain is $\varepsilon^{1}$ that reads (owing to Eq. (5)):

$$
\varepsilon^{1}=\varepsilon_{y}\left(\boldsymbol{u}^{2}\right)+\varepsilon_{x}\left(\boldsymbol{u}^{1}\right)
$$


Owing to Eq. (25), the displacement $\boldsymbol{u}^{2}$ can be read:

$$
\boldsymbol{u}^{2}=\boldsymbol{u}^{Q}-\frac{\partial \boldsymbol{u}^{1}}{\partial x_{k}} y_{k}-\frac{1}{2} \nabla_{x}^{2} \boldsymbol{U}:(\boldsymbol{y} \otimes \boldsymbol{y})
$$

Replacing in Eq. (33) the displacements $\boldsymbol{u}^{1}$ and $\boldsymbol{u}^{2}$ by Eq. (15) and Eq. (34) respectively, we deduce after some elementary manipulations:

$$
\varepsilon^{1}=\varepsilon^{Q}-\frac{\partial \varepsilon^{L}}{\partial x_{k}} y_{k}
$$

By multiplying the above expression by the elastic tensor $\mathbb{C}(\boldsymbol{y})$, we obtain a similar expression for the stress field:

$$
\boldsymbol{\sigma}^{1}=\boldsymbol{\sigma}^{Q}-\frac{\partial \boldsymbol{\sigma}^{L}}{\partial x_{k}} y_{k}
$$

In the previous relations, the solution of the problem with QBC provides the strain $\varepsilon^{Q}$ and the stress $\boldsymbol{\sigma}^{Q}$ while $\varepsilon^{1}$ and $\boldsymbol{\sigma}^{1}$ are the first correctors to the strain and the stress obtained by the asymptotic expansion method. As obvious from formula, the correctors are obtained by eliminating from $\varepsilon^{Q}$ and $\sigma^{Q}$ an elastic part represented by the tensors $\left(\partial \boldsymbol{\varepsilon}^{L}\right) /\left(\partial x_{k}\right) y_{k}$ and $\left(\partial \boldsymbol{\sigma}^{L}\right) /\left(\partial x_{k}\right) y_{k}$ in order to keep only the gradient effects. Hence, the solution of the unit cell problem with the QBC cannot be interpreted as the first corrector.

Further, as previously discussed, the first corrector vanishes as soon as the material is homogeneous at the local scale preventing spurious persistence of strain-gradient effects at the macroscopic scale. However, neither $\varepsilon^{Q}$ nor $\sigma^{Q}$ are null for a homogeneous material. To understand what is at work, consider the case of a homogeneous material in the unit cell. In such a case, tensor $\mathbb{C}(\boldsymbol{y})$ is constant, its spatial dependence with respect to $\boldsymbol{y}$ drops down in the notation and can be just denoted $\mathbb{C}$. The solution at zeroth-order, corresponding to linear boundary condition (17), is $\varepsilon^{L}=\boldsymbol{E}$ and the stress $\boldsymbol{\sigma}^{L}=\mathbb{C}: \boldsymbol{E}$. The solution with applied macroscopic gradient of strain at first-order must satisfy the QBC (28) and can then be taken into the form:

$$
\boldsymbol{u}^{Q}=\frac{1}{2} \nabla_{x}^{2} \boldsymbol{U}:(\boldsymbol{y} \otimes \boldsymbol{y}), \quad \forall \boldsymbol{y} \in \omega
$$

everywhere in the unit cell. The associated strain and stress fields are $\varepsilon^{Q}=$ $\nabla_{x} \boldsymbol{E} . \boldsymbol{y}$ and $\boldsymbol{\sigma}^{Q}=\mathbb{C}: \nabla_{x} \boldsymbol{E} . \boldsymbol{y}$, respectively. It is observed that the stress is linear with the coordinate $\boldsymbol{y}$. Getting back to the linear momentum equation (21), it can directly be verified that the former stress is statically admissible as soon as a constant body force term $\operatorname{div}_{x}\left(\boldsymbol{\sigma}^{\prime}\right)$ with $\boldsymbol{\sigma}^{L}=\mathbb{C}: \boldsymbol{E}$ is considered. This particular case illustrates the importance of the body force term in the equilibrium equation (21), without it the first-order cell problem would not have $\varepsilon^{Q}=\nabla_{x} \boldsymbol{E} \cdot \boldsymbol{y}$ as trivial solution. Now, injecting the solutions $\varepsilon^{L}=\boldsymbol{E}$ and $\varepsilon^{Q}=\nabla_{x} \boldsymbol{E} \cdot \boldsymbol{y}$ in Eq. (35), leads to the vanishing of $\boldsymbol{\varepsilon}^{1}$ and $\boldsymbol{\sigma}^{1}$. The physical 
requirement that first correctors are null for local homogeneous materials is hence satisfied.

Note that without the body force " $\operatorname{div}_{x}\left(\sigma^{Q}\right)$ ", the equilibrium equation reduces to:

$$
C_{i j k l} E_{k l, j}=0
$$

which suggests that all the components of the macroscopic strain-gradient cannot be chosen arbitrarily.

Introducing the localization rules (18) and (32) in the expression (35) leads to:

$$
\boldsymbol{\varepsilon}^{1}=\left[\mathbb{A}^{Q}(\boldsymbol{y})-\mathbb{A}^{L}(\boldsymbol{y}) \otimes \boldsymbol{y}\right] \odot_{3} \nabla_{x} \boldsymbol{E}
$$

which also gives the equality:

$$
\mathbb{A}^{1}(\boldsymbol{y})=\mathbb{A}^{Q}(\boldsymbol{y})-\mathbb{A}^{L}(\boldsymbol{y}) \otimes \boldsymbol{y}
$$

As a consequence, the components of strain-gradient elastic tensors can be determined from Eq. (13), in which the localization tensor $\mathbb{A}^{0}(\boldsymbol{y})$ is replaced by $\mathbb{A}^{L}(\boldsymbol{y})$ and $\mathbb{A}^{1}(\boldsymbol{y})$ is replaced by $\mathbb{A}^{Q}(\boldsymbol{y})-\mathbb{A}^{L}(\boldsymbol{y}) \otimes \boldsymbol{y}$.

\section{Application to a composite with fibers}

As an illustration purpose, we consider a composite with fibers. The fibers are aligned in the $x_{3}$ direction and are periodically distributed in the $\left(x_{1}, x_{2}\right)$ plane. By $R$ we denote the radius of the fiber and by $h$ the distance between two neighboring fibers (the period). The strain-gradient elastic properties are determined by considering the two kind of boundary conditions, periodic and quadratic. When the periodic boundary conditions are used, the solution is computed with the FFT method provided in [46]. The solution with quadratic boundary conditions is computed with the Finite Element Method (FEM). The elastic coefficients of the matrix are denoted by $E_{1}, \nu_{1}$ and those of the inclusions are denoted $E_{2}$ and $\nu_{2}$. The following values are considered for the numerical applications:

$$
E_{1}=1 \mathrm{GPa}, \quad E_{2}=100 \mathrm{GPa}, \quad \nu_{1}=\nu_{2}=0.3
$$

\subsection{Basic results with periodic boundary conditions}

The solution for a composite with fibers has been provided in Tran et al. [46]. In this work, the computations have been performed for one irreducible unit cell. More recently, in Barboura and Li [9], the authors claim that the strain 
gradient elastic coefficients depend on the choice of the unit cell. Particularly, they depend on the number of irreducible cells considered for their evaluation. In other words, if the calculations are performed on a unit cell containing 1,4 , $9,16 \ldots$ irreducible cells and with periodic boundary conditions, they lead to different values for the strain gradient elastic coefficients. Our results show that the strain gradient elastic response is independent of the number of periods used for the computation of the strain gradient elastic coefficients.

In order to avoid any confusion, the unit cell is still denoted $\omega$, its dimension along each space direction is still denoted by $h$. The unit cell can contain multiple periods, i.e. multiple irreducible unit cells denoted $\omega_{0}$ and having the dimension $h_{0}$. In Fig. 2, we show three unit cells containing 1, 4, 9 irreducible cells.

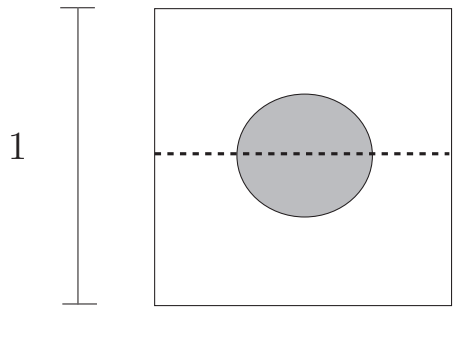

$1 \times 1$

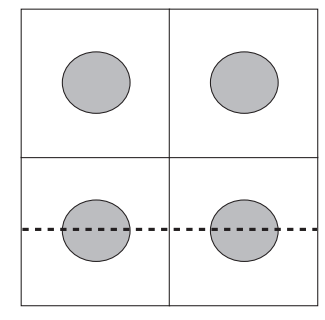

$2 \times 2$

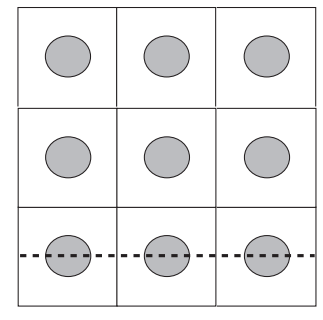

$3 \times 3$

Fig. 2. Unit cells for the considered numerical computations.

Considering first the case of classic homogenization, the macroscopic elastic coefficients are determined by computing the solution of the first order cell problem given by Eq. (7). These coefficients remains unchanged if we consider one or multiple periods for $\omega$. It can be easy proved. Note first that the rapid variable $\boldsymbol{y}$ is obtained by dividing the true space variable $\boldsymbol{\chi}$ by the dimension of the unit cell $h$. As a consequence, for a 2D problem with a squared unit cell, the non dimensional variable vary from -0.5 to 0.5 along each space direction, and this whatever the number of periods considered in the unit cell ( $\omega$ can contain 1,4,9... irreducible cells). Consider an irreducible cell which paves the unit cell $\omega$ and let us denote by $\boldsymbol{Y}_{0}$ the position of its center. We introduce the following coordinate change variable $\overline{\boldsymbol{y}}=N\left(\boldsymbol{y}-\boldsymbol{Y}_{0}\right)$ where $N$ is the number of unit cells considered along each space direction. With this definition, the variable $\overline{\boldsymbol{y}}$ vary from -0.5 to 0.5 in the irreducible cell. The two space variables $\boldsymbol{y}$ and $\overline{\boldsymbol{y}}$ are illustrated in Fig. (3). 


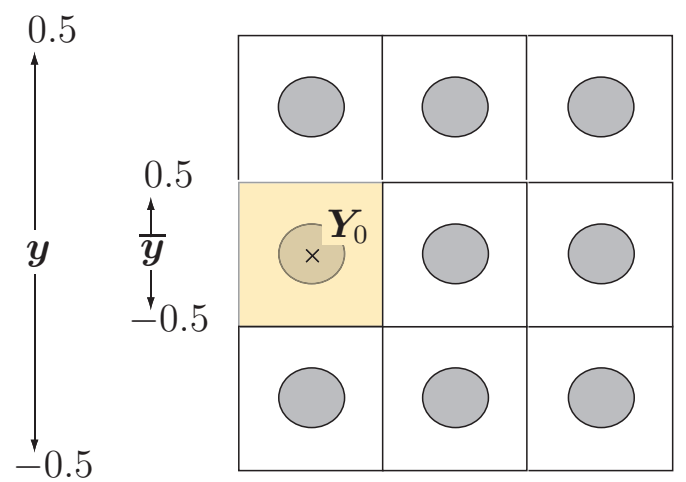

Fig. 3. Definition of the two coordinates $\boldsymbol{y}$ and $\overline{\boldsymbol{y}}$. $\boldsymbol{y}$ lies in the unit cell $\omega$, and $\overline{\boldsymbol{y}}$ lies in an irreducible unit cell $\omega_{0}$.

By applying in the first order cell problem given by Eq. (7) the following change of variables:

$$
\begin{array}{r}
\boldsymbol{u}^{1}(\boldsymbol{x}, \boldsymbol{y})=\frac{1}{N} \overline{\boldsymbol{u}}^{1}(\boldsymbol{x}, \overline{\boldsymbol{y}}) \\
\varepsilon^{0}(\boldsymbol{x}, \boldsymbol{y})=\overline{\boldsymbol{\varepsilon}}^{0}(\boldsymbol{x}, \overline{\boldsymbol{y}}) \\
\boldsymbol{\sigma}^{0}(\boldsymbol{x}, \boldsymbol{y})=\overline{\boldsymbol{\sigma}}^{0}(\boldsymbol{x}, \overline{\boldsymbol{y}}) \\
\boldsymbol{y}^{\prime}=N\left(\boldsymbol{y}-\boldsymbol{Y}_{0}\right)
\end{array}
$$

we obtain:

$$
\left\{\begin{array}{l}
\operatorname{div}_{\bar{y}}\left(\overline{\boldsymbol{\sigma}}^{0}(\boldsymbol{x}, \overline{\boldsymbol{y}})\right)=0 \\
\overline{\boldsymbol{\sigma}}^{0}(\boldsymbol{x}, \overline{\boldsymbol{y}})=\mathbb{C}(\overline{\boldsymbol{y}}): \overline{\boldsymbol{\varepsilon}}^{0}(\boldsymbol{x}, \overline{\boldsymbol{y}}) \\
\overline{\boldsymbol{\varepsilon}}^{0}(\boldsymbol{x}, \overline{\boldsymbol{y}})=\boldsymbol{\varepsilon}_{\bar{y}}\left(\overline{\boldsymbol{u}}^{1}(\boldsymbol{x}, \overline{\boldsymbol{y}})\right)+\boldsymbol{E}(\boldsymbol{x}) \\
\boldsymbol{\varepsilon}_{\bar{y}}\left(\boldsymbol{u}^{1}(\boldsymbol{x}, \overline{\boldsymbol{y}})\right)=\frac{1}{2}\left(\nabla_{\bar{y}} \boldsymbol{u}^{1}(\boldsymbol{x}, \overline{\boldsymbol{y}})+\nabla_{\bar{y}}^{T} \overline{\boldsymbol{u}}^{1}(\boldsymbol{x}, \overline{\boldsymbol{y}})\right)
\end{array}\right.
$$

The equations of the problem then remain unchanged. This confirms that the strain and the stress computed with one or multiple unit cells remain unchanged. As for example, we show in Fig. 4 the distribution of the stress component $\sigma_{11}^{0}$, due to the macroscopic strain $E_{11}=1$, along a horizontal line which passes through the centers of the inclusions (see the dashed line in Fig. 2 ) in the case of 1, 4 and 9 irreducible cells. It is observed that the solution remains the same for each case. The solution for multiple periods is obtained by the repetition of the solution obtained with one irreducible cells. 

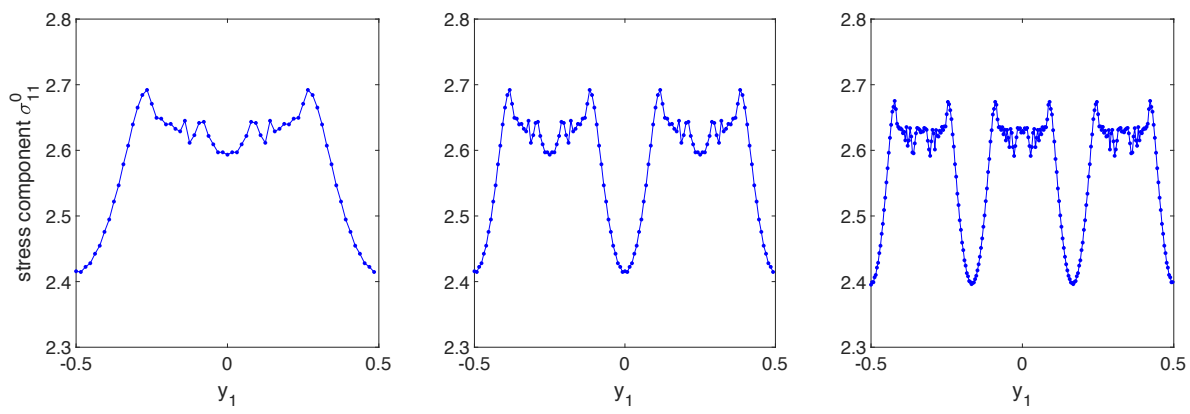

Fig. 4. Distribution of the stress component $\sigma_{11}^{0}$, due to the macroscopic strain $E_{11}=1$, along a horizontal line which passes through the centers of the inclusions.

As consequence, the classic homogenized elastic coefficients (components of $\mathbb{C}^{0,0}$ ) also remain unchanged if we make the computations with one or multiple periods.

Now, if we consider the higher order problem given by Eq. (10) in which we apply the change of variable (42)-(45) for the displacement $\boldsymbol{u}^{1}$, the stress $\boldsymbol{\sigma}^{0}$ and the position vector $\boldsymbol{y}$, we obtain:

$$
\left\{\begin{array}{l}
N \operatorname{div}_{\bar{y}}\left(\boldsymbol{\sigma}^{1}(\boldsymbol{x}, \overline{\boldsymbol{y}})\right)+\operatorname{div}_{x}\left(\overline{\boldsymbol{\sigma}}^{0}(\boldsymbol{x}, \overline{\boldsymbol{y}})-<\overline{\boldsymbol{\sigma}}^{0}(\boldsymbol{x}, \overline{\boldsymbol{y}})>_{\omega}\right)=0 \\
\boldsymbol{\sigma}^{1}(\boldsymbol{x}, \overline{\boldsymbol{y}})=\mathbb{C}(\overline{\boldsymbol{y}}): \boldsymbol{\varepsilon}^{1}(\boldsymbol{x}, \overline{\boldsymbol{y}}) \\
\boldsymbol{\varepsilon}^{1}(\boldsymbol{x}, \overline{\boldsymbol{y}})=N \boldsymbol{\varepsilon}_{\bar{y}}\left(\boldsymbol{u}^{2}(\boldsymbol{x}, \overline{\boldsymbol{y}})\right)+\frac{1}{N} \varepsilon_{x}\left(\overline{\boldsymbol{u}}^{1}(\boldsymbol{x}, \overline{\boldsymbol{y}})\right) \\
\boldsymbol{\varepsilon}_{\bar{y}}\left(\boldsymbol{u}^{2}(\boldsymbol{x}, \overline{\boldsymbol{y}})\right)=\frac{1}{2}\left(\nabla_{\bar{y}} \boldsymbol{u}^{2}(\boldsymbol{x}, \overline{\boldsymbol{y}})+\nabla_{\bar{y}}^{T} \boldsymbol{u}^{2}(\boldsymbol{x}, \overline{\boldsymbol{y}})\right) \\
\boldsymbol{\varepsilon}_{x}\left(\overline{\boldsymbol{u}}^{1}(\boldsymbol{x}, \overline{\boldsymbol{y}})\right)=\frac{1}{2}\left(\nabla_{x} \overline{\boldsymbol{u}}^{1}(\boldsymbol{x}, \overline{\boldsymbol{y}})+\nabla_{x}^{T} \overline{\boldsymbol{u}}^{1}(\boldsymbol{x}, \overline{\boldsymbol{y}})\right)
\end{array}\right.
$$

It is observed that the problem remains unchanged for 1 unit cell or multiple unit cells if we apply the following change of variables for $\boldsymbol{u}^{2}, \varepsilon^{1}$ and $\boldsymbol{\sigma}^{1}$ :

$$
\begin{array}{r}
\boldsymbol{u}^{2}(\boldsymbol{x}, \boldsymbol{y})=\frac{1}{N^{2}} \overline{\boldsymbol{u}}^{1}(\boldsymbol{x}, \overline{\boldsymbol{y}}) \\
\varepsilon^{1}(\boldsymbol{x}, \boldsymbol{y})=\frac{1}{N} \overline{\boldsymbol{\varepsilon}}^{1}(\boldsymbol{x}, \overline{\boldsymbol{y}}) \\
\boldsymbol{\sigma}^{1}(\boldsymbol{x}, \boldsymbol{y})=\frac{1}{N} \overline{\boldsymbol{\sigma}}^{1}(\boldsymbol{x}, \overline{\boldsymbol{y}})
\end{array}
$$

As a consequence, the strain $\varepsilon^{1}$ and the stress $\sigma^{1}$ are the same for multiple periods but their amplitudes are divided by a factor $N$. This is confirmed in Fig. 5. The distribution of the stress component $\sigma_{11}^{1}$ due to a prescribed macroscopic gradient of strain $E_{11,1}=1$ is given for 1,4 and 9 irreducible cells. It is observed that the amplitude of the stress is divided by a factor 2 in the case of a unit cell with 4 irreducible cells and by a factor 3 for 9 irreducible cells. 

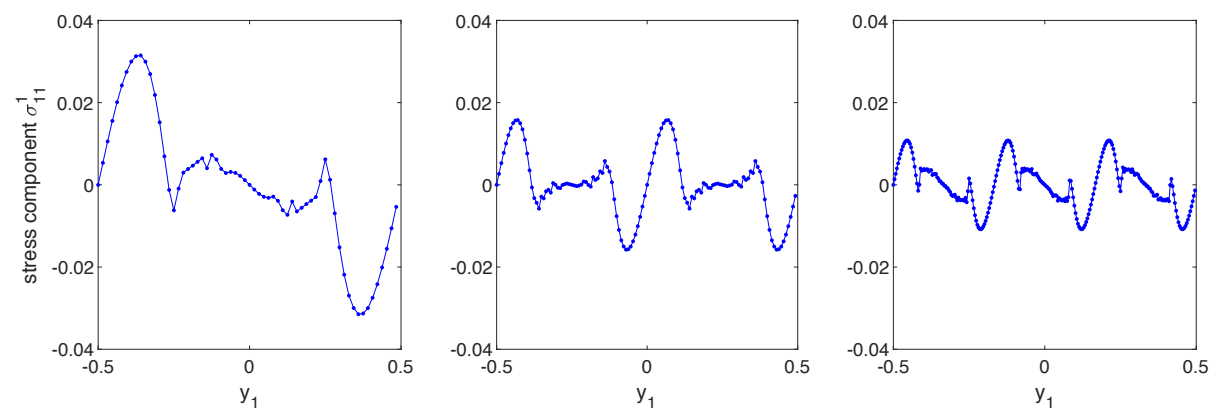

Fig. 5. Distribution of the stress component $\sigma_{11}^{1}$, due to the macroscopic strain $E_{11,1}=1$, along a horizontal line which passes through the centers of the inclusions.

Since the strain $\varepsilon^{1}$ and the stress $\sigma^{1}$ are divided by a factor $N$ in the case of a unit cell containing $N \times N$ irreducible cells, the corresponding energy is divided by a factor $N^{2}$. As a consequence the sixth order elastic tensor $\mathbb{C}^{1,1}$ is divided by a factor $N^{2}$. However, let us recall that the relation between the hyperstress and the macroscopic gradient of strain involves the term $h^{2} \mathbb{C}^{1,1}$ in which $h=N h_{0}, h_{0}$ being the dimension of the irreducible unit cell. As a consequence, the quantity $h^{2} \mathbb{C}^{1,1}=h_{0}^{2} N^{2} \mathbb{C}^{1,1}$ is independent of the number of periods and can be determined by considering only one irreducible cell. For instance, we provide in Fig. 6 the value of $C_{111111}^{1,1}$ as function of the number of periods. It is observed that the component $C_{111111}^{1,1}$ decreases with the number of periods while the quantity $N^{2} C_{111111}^{1,1}$ is independent.

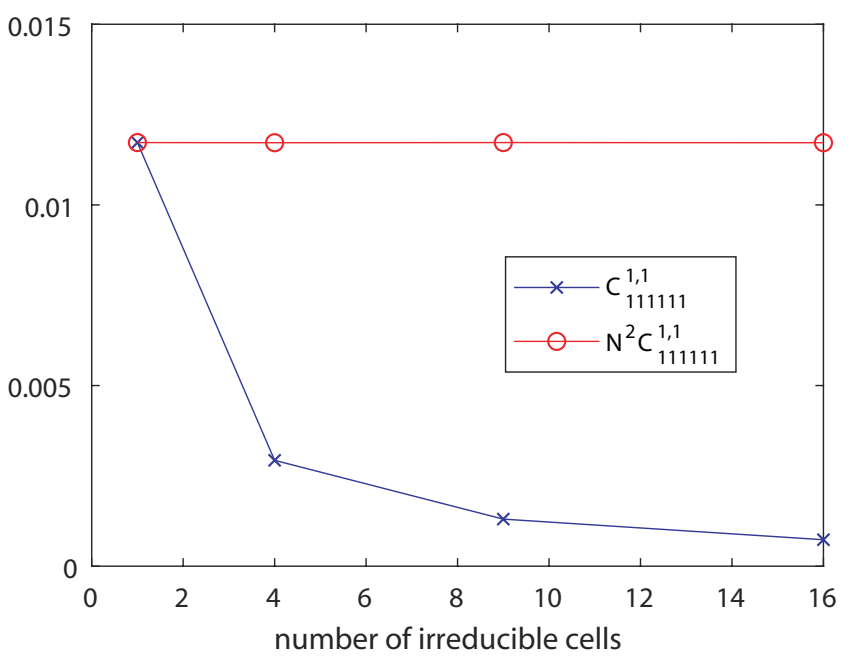

Fig. 6. Variations of $C_{111111}^{1,1}$ and $N^{2} C_{111111}^{1,1}$ with the number of periods $N$. 


\subsection{Comparison between $P B C$ and $Q B C$}

When the QBC is used, the results for the homogenized elastic properties depend on the number of periods. The FEM-based approach uses the correction with the body force " $\operatorname{div}_{x}\left(\boldsymbol{\sigma}^{L}\right)$ ". The effective strain-gradient elasticity properties are computed with the modified average rule (13). The results are provided only for the component 111111 of the sixth-order strain-gradient elasticity tensor and are given in Fig. 7. The variations of the strain gradient elastic component are given as function of the radius of the fiber. The result is compared with that obtained with the periodic boundary condition (and computed with the FFT). It can be observed in Fig. 7 that for $R=0$ the component of the strain-gradient elastic coefficient is null, this result clearly shows that the new method eliminates the persistence of spurious strain-gradient effects. It can also be observed that the FEM solution depends on the number of irreducible unit cells in the RVE and, by increasing their number, that the QBC-based solutions converge to FFT-based one. One explanation is that, considering the Saint-Venant principle, the solution at distance from the boundary of the RVE is independent of the choice of the kind of boundary conditions. For a RVE constituted of a large number of irreducible cells, the periodicity is almost recovered for the cells in the bulk. Since there is a high number of strain gradient elastic coefficients, we only present the results for one component. Other coefficients have been computed with the same conclusion: (i) no persistence when the inclusion radius return to zero with $\mathrm{QBC}$ and $\mathrm{PBC}$, (ii) the solution with $\mathrm{QBC}$ tends to the solution with $\mathrm{PBC}$ when we increase the number of irreducible cells. If we deal with periodic composites, the method based on PBC is more accurate. Indeed, the homogenized elastic coefficients are determined by the computations over one irreducible cell while, with the QBC, it requires the computation on multiple periods and the number of irreducible cells must be increased in order to obtain the convergence. Obviously, this allows higher computational requirements. However, the scope of the present paper is to develop a new method based on QBC to deal with non-periodic microstructures. No examples have been provided in the present paper but applications to the case of non-periodic microstructures have to be considered in a future work. 


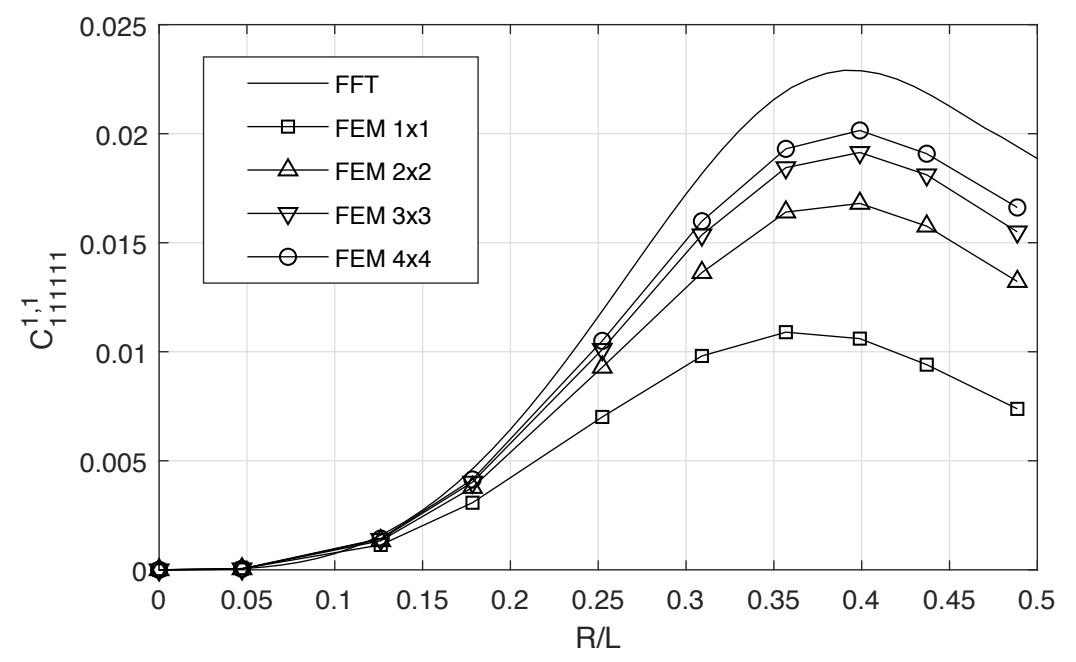

Fig. 7. Variation of the strain-gradient elastic coefficient $C_{111111}^{1,1}$ as function of the ratio $R / L$. Comparison between the solution with $\mathrm{PBC}(\mathrm{FFT})$ and $\mathrm{QBC}(\mathrm{FEM})$.

For comparison purpose, we also provide the strain-gradient elastic coefficient computed by the FEM on an irreducible unit cell with the older definition for the elastic energy. The results are provided in Fig. 8 by accounting for the constant body force " $\operatorname{div}_{x}\left(\boldsymbol{\sigma}^{L}\right)$ " and in Fig. 9 without the body force. First, in both cases (with or without the body force), the persistence of the straingradient elasticity is observed when the radius of the fibre is $R=0$. It must be also noted that, comparatively to the results provided on Fig. 7, the straingradient coefficients computed with the older method largely overestimate the strain-gradient effects. Additionally, the presence of the body force in the equilibrium equation has a significant influence on the value of the straingradient elasticity coefficients.

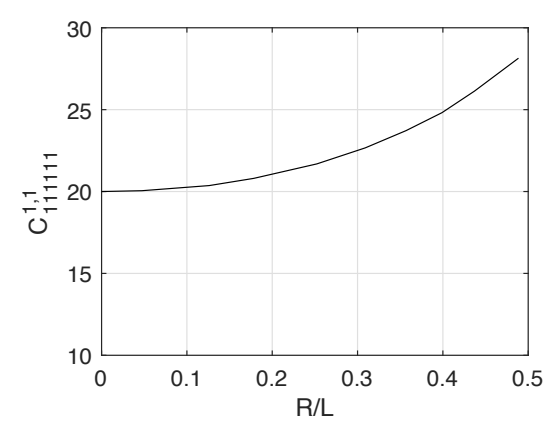

Fig. 8. Variation of the strain-gradient elastic coefficient $C_{111111}^{1,1}$ as function of the ratio $R / L$. Computation including the body force in the equilibrium equation 


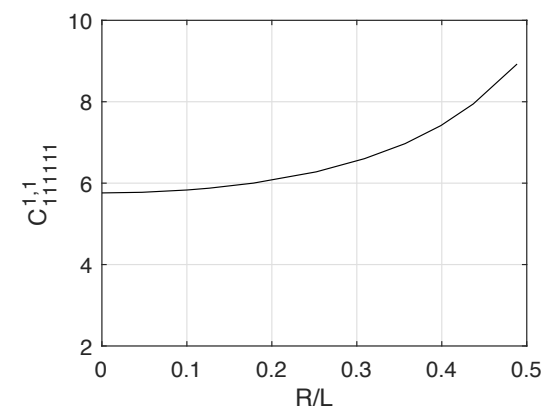

Fig. 9. Variation of the strain-gradient elastic coefficient $C_{111111}^{1,1}$ as function of the ratio $R / L$. Evaluation without considering the body force term.

\section{Conclusion}

In this paper, we have provided a unified approach to the determination of the strain-gradient elasticity properties. Two methods have been investigated. The first is based on asymptotic series expansion within the framework of periodic homogenization, the second consider the quadratic condition (QBC) on the boundary of the representative volume element (RVE). A bridge between the two methods has been established and a modification of the QBC-based method has been provided in order to be consistent with the periodic homogenization. The main significant result of the paper is that the new method based on QBC allows to eliminate the persistence of the strain-gradient effects: for a homogeneous material at the local scale the macroscopic description remains of Cauchy type, so that all the components of the strain-gradient elastic tensor must be equal to zero. Strain-gradient effects only appears when the material is heterogeneous at the local scale. This results has been confirmed by the numerical implementation of the two methods in the particular case of a composite material with fibers.

\section{References}

[1] H. Abdoul-Anziz and P. Seppecher. Homogenization of periodic graphbased elastic structures. Journal de l'Ecole Polytechnique-Mathématiques, 2018.

[2] H. Abdoul-Anziz and P. Seppecher. Strain gradient and generalized continua obtained by homogenizing frame lattices. Mathematics and mechanics of complex systems, 6(3):213-250, 2018.

[3] J.-J. Alibert, P. Seppecher, and F. Dell'Isola. Truss modular beams with 
deformation energy depending on higher displacement gradients. Mathematics and Mechanics of Solids, 8(1):51-73, 2003.

[4] G. Allaire. Homogenization and two-scale convergence. SIAM Journal on Mathematical Analysis, 23(6):1482-1518, 1992.

[5] J.L. Auriault and E. Sanchez-Palencia. Study of macroscopic behavior of a deformable porous medium. J. Mécanique., 16(4):575-603, 1977.

[6] M. Bacca, D. Bigoni, F. Dal Corso, and D. Veber. Mindlin second-gradient elastic properties from dilute two-phase Cauchy-elastic composites. Part I: Closed form expression for the effective higher-order constitutive tensor. Int. J. Solids Structures, 50:4010-4019, 2013.

[7] M. Bacca, D. Bigoni, F. Dal Corso, and D. Veber. Mindlin second-gradient elastic properties from dilute two-phase Cauchy-elastic composites. Part II: Higher-order constitutive properties and application cases. Int. J. Solids Structures, 50:4020-4029, 2013.

[8] N. Bakhvalov and G. Panasenko. Homogenization: Averaging processes in periodic media. 1989.

[9] S. Barboura and J. Li. Establishment of strain gradient constitutive relations by using asymptotic analysis and the finite element method for complex periodic microstructures. Int. J. Solids and Structures, 136137:60-76, 2018.

[10] A. Bensoussan, J.-L. Lions, and G.C. Papanicolaou. Asymptotic Analysis for Periodic Structures. North-Holland, 1978.

[11] D. Besdo and H.-U. Dorau. Inelastic behaviour of plane frictionless blocksystems described as Cosserat media. Arch. Mech., 65:124-125, 1985.

[12] D. Besdo and H.-U. Dorau. Zur Modellierung von Verbundmaterialien als homogenes Cosserat - Kontinuum. Z. Angew. Math. Mech., 68:153-155, 1988.

[13] D. Bigoni and W.J. Drugan. Analytical derivation of Cosserat moduli via homogenization of heterogeneous elastic materials. J. Appl. Mech., 74:741-753, 2007.

[14] C. Boutin. Microstructural effects in elastic composites. Int. J. Sol. Struct., 33(7):1023-1051, 1996.

[15] C. Boutin. Homogenization Methods and Generalized Continua in Linear Elasticity. Altenbach H., Oechsner A. (eds) Encyclopedia of Continuum Mechanics. Springer., 2019.

[16] C. Boutin and J. Soubestre. Generalized inner bending continua for linear fiber reinforced materials. Int. J. Sol. Struct., 48:517-534, 2011.

[17] F. Bouyge, I. Jasiuk, and M. Ostoja-Starzewski. A micromechanically based couple-stress model of an elastic two-phase composite. Int. J. Sol. Struct., 38:1721-173, 2001.

[18] F. Bouyge, I. Jasiuk, and M. Ostoja-Starzewski. A micromechanically based couple-stress model of an elastic orthotropic two-phase composite. Eur. J. Mech. A, 21:465-481, 2002.

[19] P.H. Dederich and R. Zeller. Variational treatment of the elastic constants of disordered materials. Zeitschrift für Physik, 259:103-116, 1973. 
[20] G. Diener, A. Hurrich, and J. Weissbarth. Bounds on the non-local effective elastic properties of composites. J. Mech. Phys. solids, 32(1):21-39, 1984.

[21] G. Diener, C.H. Raabe, and J. Weissbarth. Bounds for the non-local effective properties of random media. J. Mech. Phys. solids, 29(3):181198, 1981.

[22] G. Diener, C.H. Raabe, and J. Weissbarth. Bounds for the non-local effective properties of random media ii. J. Mech. Phys. solids, 30(5):305322, 1982.

[23] W.J. Drugan. Micromechanics-based variational estimates for a higherorder nonlocal constitutive equation and optimal choice of effective moduli for elastic composites. J. Mech. Phys. Solids, 48:1359-1387, 2000.

[24] W.J. Drugan. Two exact micromechanics-based nonlocal constitutive equations for random linear elastic composite materials. J. Mech. Phys. Solids, 51:1745-1772, 2003.

[25] W.J. Drugan and J.R. Willis. A micromechanics-based nonlocal constitutive equation and estimates of representative volume element size for elastic composites. J. Mech. Phys. Solids, 44(4):497-524, 1996.

[26] N.A. Fleck and J.W. Hutchinson. Strain gradient plasticity. Adv. Appl. Mech., 33:295-361, 1997.

[27] S. Forest. Mechanics of generalized continua: construction by homogenization. J. Phys. IV, pages 39-48, 1998.

[28] S. Forest and D.K. Trinh. Generalized continua and non-homogeneous boundary conditions in homogenisation methods. ZAMM Z. Angew. Math. Mech., 91:90-109, 2011.

[29] B. Gambin and E. Kroner. High order terms in the homogenized stressstrain relation of periodic elastic media. Phys. Stat. Sol.(b), 151:513-519, 1989.

[30] M. Gologanu, J.B. Leblond, G. Perrin, and J. Devaux. Recent extensions of Gurson's model for porous ductile metals. Continuum Micromechanics ed. P. Suquet, Springer Verlag, 1997.

[31] A.L. Gurson. Continuum theory of ductile rupture by void nucleation and growth. Part I. - Yield criterion and flow rules for porous ductile media. J. Engrg. Mater. Technology, 99:2-15, 1977.

[32] Z. Hashin and S. Shtrikman. On some variational principles in anisotropic and nonhomogeneous elasticity. J. Mech. Phys. Solids, 10(4):335-342, 1962.

[33] Z. Hashin and S. Shtrikman. A variational approach to the theory of the elastic behavior of polycrystals. J. Mech. Phys. Solids, 10:343-352, 1962.

[34] V. Kouznetsova, M.G.D. Geers, and W.A.M. Brekelmans. Multi-scale constitutive modeling of heterogeneous materials with gradient enhanced computational homogenization scheme. Int. J. Num. Meth. Eng., 54:1235-1260, 2002.

[35] V. Kouznetsova, M.G.D. Geers, and W.A.M. Brekelmans. Multi-scale second-order computational homogenization of multi-phase materials: a 
nested finite element solution strategy. Comput. Methods Appl. Mech. Engrg., 193:5525-5550, 2004.

[36] E. Kröner and H. Koch. Effective properties of disordered materials. Solid Mech. Arch., 1:183-238, 1976.

[37] R.D. Mindlin. Micro-structure in linear elasticity. Arch. Rat. Mech. Anal., 16:51-78, 1964.

[38] R.H.J. Peerlings and N.A. Fleck. Numerical analysis of strain gradient effects in periodic media. Journal de Pysique. IV :JP, 11(5):5153-5160, 2001.

[39] C. Pideri and P. Seppecher. A second gradient material resulting from the homogenization of an heterogeneous linear elastic medium. Continuum Mech. Thermodyn., 9:241-257, 1997.

[40] C. Pideri and P. Seppecher. Un résultat d'homogénéisation pour un matériau élastique renforcé périodiquement par des fibres élastiques de très grande rigidité. C.R. Acad. Sci. IIB, 324(7):475-481, 1997.

[41] E. Sanchez-Palencia. Comportements local et macroscopique d'un type de milieux physiques hétérogènes. Int. J. Engng Sci., 12:331-351, 1974.

[42] E. Sanchez-Palencia. Nonhomogeneous Media and Vibration Theory, volume 127. Springer, Berlin, 1980.

[43] V.P. Smyshlyaev and K.D. Cherednichenko. On rigorous derivation of strain gradient effects in the overall behaviour of periodic heterogeneous media. J. Mech. Phys. Solids, 48:1325-1357, 2000.

[44] P. Suquet. Plasticité et homogénéisation. PhD thesis, Université Pierre et Marie Curie, Paris VI, 1982.

[45] R. Toupin. Elastic materials with couple-stresses. Arch. Rat. Mech. Anal., 11(1):385-414, 1962.

[46] T.-H. Tran, V. Monchiet, and G. Bonnet. A micromechanics-based approach for the derivation of constitutive elastic coefficients of straingradient media. Int. J. Sol. Struct., 49(5):783-792, 2012.

[47] N. Triantafyllidis and S. Bardenhagen. The influence of scale size on the stability of periodic solids and the role of associated higher order gradient continuum models. J. Mech. Phys. Solids, 44:1891-1928, 1996.

[48] X. Yuan, Y. Tomita, and T. Andou. A micromechanical approach of nonlocal modeling for media with periodic microstructures. Mech. Res. Comm., 35:126-133, 2008.

[49] L. Zybell, U.Mühlich, and M. Kuna. Constitutive equations for porous plane-strain gradient elasticity obtained by homogenization. Arch. Appl. Mech., 79:359-375, 2009. 